\title{
A DATA DISSEMINATION MODEL FOR VEHICULAR ADHOC NETWORK
}

\author{
Anshu Garg, \\ Amity School of Engineering and Technology \\ Amity University, Sec-125, NOIDA, (U.P.), India
}

\begin{abstract}
Absract
Vehicular adhoc network (VANET) is an emerging network which is depend on the communication of data through vehicular network via data pushing. Data pushing in between the vehicles was done by the data pushing communication model. In this paper, we present a formal model of data dissemination in between the vehicles by the mobility of vehicles and describe also the affects of data dissemination and study their characteristics which define the mobility if data dissemination in bidirectional mobility, we also study the data push model in the context of Traffic View, a system that we have implemented to disseminate information about the vehicles on the road. Traffic data could be disseminated using the cars moving on the same direction, cars moving in the opposite direction, or cars moving in both directions.
\end{abstract}

Keywords: adhoc, communication, broadcasting, network, traffic.

\section{Introduction}

In future, number of vehicles are equipped with the communicating device commonly that may be known as telematics and this telematics can help to communicate between all the vehicles. For instance, it is predicted that the number of telematics subscribers in the United States will reach more than 15 million by 2009 [5]. Inter vehicle communication becoming so important research field in promising the vision of intelligent transport system [3]. Such type of system can enable all the applications like communication between the vehicles, collision avoidance and its recovery, emergency passing of data over the network, dynamic route scheduling and it will also helps to find all the reporting between the road accident and all the circumstances or incident that are taken on the network of the communication and communicates in between all the vehicles via cellular networks and the vehicle networks.

Vehicular adhoc network is emerging a preferred network for intelligent transport system. Vehicular adhoc network is a network wireless network having very short range of communication i.e. IEEE802.11 between vehicles. The range given by the federal communication commissions has $75 \mathrm{MHz}$ in the $5.9 \mathrm{Ghz}$ band for the licensed dedicated short range communication [4]. The main aim of the

\author{
Prof.(Dr.) Sanjeev Bansal \\ Amity School of Engineering and Technology \\ Amity University, Sec-125, NOIDA, (U.P.), India
}

communication was to enhance the bandwidth and the reducing latency for vehicle to vehicle and vehicle to infrastructure communication. The adoption of Dedicated Short Range Communication spectrum between the vehicle to vehicle communication for increasing the interest and expectation from the new technology. Other networks like cellular and mobile networks are constructed on the fly and not require the wireless network interface, which will be a standards features for the next generation of vehicles. Furthermost, vehicular adhoc network enables new class applications and also takes time critical response or high data transfer rates. The main problem in the vehicular adhoc networks is that How to exchange traffic information among vehicles. In this applications the data is dissiminated into the broadcast-based application. In this approach the potential of bootstrapping vehicular adhoc network. In this paper we focus on the data push communication model in vehicular adhoc network. The main approach for the data push communication is to exchange the information like the position of the vehicle and the speed of the vehicle among the set of moving vehicles in order to enable the each individual vehicle to assess and view all the conditions in front of it. The main mechanism can be used to achieve the goal like flooding and dissemination. In flooding mechanism, each individual vehicles broadcast all the information as they like there is no other meaningful information was sent by the other one. Every time the vehicle receives the message and store this message immediately and forward this message to rebroadcating the message to the other vehicle. This mechanism is not scalable due to large number of messages flooded over the network in very high congested network. In the dissemination mechanism, each vehicle broadcast information to all the vehicles and shared all the information to all of the vehicles and also save the information and updates timely the updated broadcast information. This dissemination mechanism is high scalable, so the number of broadcast messages is limited and they do not flood the networks. This dissemination mechanism can either broadcast information to the vehicular networks in all direction, or perform a directed network between all the vehicles. Furthermost, the vehicular dissemination model is very useful to communication between all the vehicles either the vehicle in the same direction or in bidirectional, either is the same path or the different. Further the vehicles 
communication could be relayed using omly the vehicles travelling in the direction of the vehicles travelling in the same direction or in the other directions, or the vehicles travelling in the both directions. To decide that the best dissemination model, a formal model must requires the traffic parameters for the vehicular networks.

This paper present the formal model of data dissemination I vehicular adhoc network and analyzes how it characteristics, mainly for the bidirectional mobility of vehicles in definite paths, that affects all ht performance of data dissemination. We evaluate, by means of simulation, three data dissemination models: same - dir, opp - dir, and bi - dir in the context of Traffic View [21, $10]$, a system for scalable traffic data dissemination and visualization in VANETs.

\section{Traffic View}

Traffic View is a system for traffic data dissemination and visualizes all the information for the vehicular adhoc networks. The main goal of the traffic view is to provide the continuous updates about the traffic control, which can contained all the traffic information and given to the driver for the route planning as well as driving updates either for the weather information or the network information for the vehicles.

\subsection{Traffic Overview:-}

All the Vehicle are running in equipped with a computing device which contains a short range wireless interface to communicate with each other that may be known as GPS Receiver i.e. Gross Docket Services optionally, an on board diagnostics system (OBD) Interface is used in Vehicle which require all the configuration like electrical data $\&$ mechanical device helps to sensing the vehicles \& these sensors are installed in vehicles. The GPS receiver provides all the facilities to the vehicles like location, current time, speed, location of station \& direction of the vehicle. Each Participating vehicle gather information from all the other vehicles \& station also. This Approach was known as peer to peer communication $b / w$ the vehicle N/W. The display shows a map connected to other vehicles and annonated with real time condition for traffic $\&$ contained dynamic information about the vehicles around them such like location, Each vehicle in this N/W contained all the information \& stored all the information of all vehicles into the database. The records of the distance are periodically broadcasted \& contain all the update information. A vehicle contain the record consist of vehicle identification, position of vehicle in the form of latitude and longitude, correct speed time \& all the time stamp parameter of the vehicle was created \& the maintained record was created. In traffic view, we have chosen to broadcast all the data stored in a vehicle of a single packet. This sample data propagation model contained the three advantage they are:-
1. It limit all the bandwidth consumed by each of the vehicle.

2. It limit all the number of re-transmission of the data due to collision.

\section{It avoid all the dealing with flow control.}

The flow control mechanism is necessary if data would be split in multiple Packets.

The data stored in the vehicle is usually greater than the size of the packet contained. Therefore, the data aggregation approach is used to the applied reco5d exchanged. Data aggregation is an approach which depends on the semantics of the data. Data aggregation approach is used in ratio based mechanism through which the front of vehicle is divided into number of regions $(1<=\mathrm{i}<=\mathrm{n})$. For each of the region the ai is assigned which is increase of the number of the individual record that would be aggregated. Each region is also assigned to the portion $(0<\mathrm{pi}<=1)$ where, pi is the remaining free space in the broadcast message.

\subsection{Traffic View Prototype}

In prototype, we will developed a working prototype of traffic view which will be installed in the vehicles and tested by the out door ones under the traffic or real condition. We will evaluated our prototype by means of practical's which was performed under the traffic view system or in a highway environment. In this process, we have cover all the circumstances and deployment of the particular vehicular application. This traffic new prototype was applied or implemented mostly in the java or c languages that have been operated in both the windows and linux. The graphics may be designed and developed in openGL for the better picture resolution and graphics display, through which the user interface was so much high because the interface must contained the two pamel are: Near-View and Map-View. The near-view describes all the vehicles that are being on the road are displayed. The map-view display the map which contained all the information about cars. Both of these views are defined publicly through which they may be represented. In order to clear all the GPS in accuracy, that we also given an algorithm for the cars through which the car should be moved. The above figure presents the traffic flow prototype that was installed in the car. As the above picture in the figure, we have attached an antenna on a car with $802.11 \mathrm{~b}$ data card. During the initial real life traffic experiment on the road, we realized that having the wireless card inside the car decrease the communication range significantly. Here, there was an omni directional antenna above the car when increase the communication range between 0-300metre. 


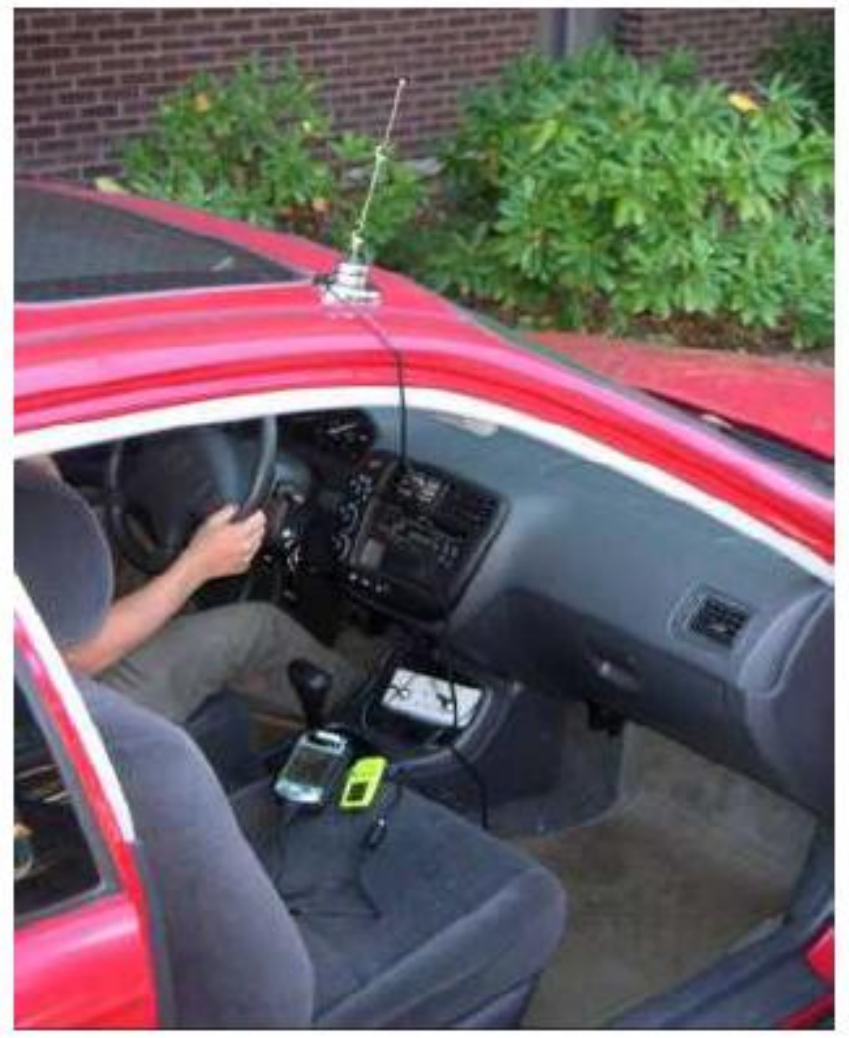

Fig-1

On the another side, we have observed that the speed does not influence any system within the limited number of cars. In which the traffiv view system, the simulator is used to design this type of services like ns-2 means network simulator, which define all the characteristics of the communication. Data communication can be defined all the data transfer and read. Here, some arrgregation algorithm are used that have evaluated and compares all of these using simulation environment $[21,10,22]$. This paper was also used in the traffic view simulation environment to compare the efficiency and effectiveness of data dissemination approach for the vehicular adhoc network.

\section{Analysis of Data Dissemination in Traffic View}

In which we can describe and anlyze of the different dissemination moel assumption and designing approach for dissemination model.

\subsection{Model Assumptions}

In the analysis of data dissemination model we assume that the vehicles are moving on the bidirectional road or the highway or on the other roads where each direction of the highway connect multiple lanes that is represented in figure-2. We assume that the movement of direction to be either right as shown in the lower part of the figure-2 like $v 1 r$ and $v 2 r$ shown in the left upper part of the figure. The vehicle average speed of sr and sl for the right and left directions respectively. We assumed that the vehicles are equipped with some system techniques like GPS and the wireless devices and the external antenna which can communicate in omni-directional approach and having the communication rangeR1. In traffic view, each vehicle in the road can give all the information of the road vehicle to maintain vehicle information and road information should be propogated with respect to their major direction either in single in opposite direction. in broadcasting networks the data packets are sent after B seconds that containing no loss of granularity and for the sake of simplicity and propogating all the information that the vehicle are moving either in left or right direction.

\subsection{Dissemination Models}

In dissemination model we can describe the difference between the two types of broadcast data like generated data and the relayed data. In generated data it can store the information speed and location on the vehicle or the network and it may be shown by small red circle. In relayed data it can store the data and the broadcast data of the information data and it may be showed as the large yellow circle.

In this paper we can improve the difference between the maindissemination model and propogaton model like some direction, opposite direction and bi dierection which is the original TrafficView model, each vehicle broadcasts both its generated data and the relayed data in a single packet. This broadcasted information propagates only through vehicles moving in the same direction. Therefore, a vehicle drops any received packet broadcasted by another vehicle on the same road behind it. More specifically, when a vehicle v1 broadcasts a data packet; vehicle v2 will accept this packet if and only if:

1. $\mathrm{v} 2$ is within the transmission range of $\mathrm{v} 1$, and

2. v1 and $\mathrm{v} 2$ are moving in the same direction, and

3. $\mathrm{v} 1$ is in front of $\mathrm{v} 2$ with respect to their movements.

Figure 2(a) shows how information is propagated from vehicle v1R to vehicle v5R in the Right direction using

same-dir model. Note that no vehicle from the opposite direction participates in this model. On the other hand, in opp-dir model, vehicles in same direction (e.g., Right) only broadcast the generated data. These generated data are accumulated and propagated backwards by the vehicles in the opposite direction (e.g., Left). When vehicle v1 broadcasts a packet, v2 which is within the transmission range of $\mathrm{v} 1$ will handle the received packet as follow: 
(a)

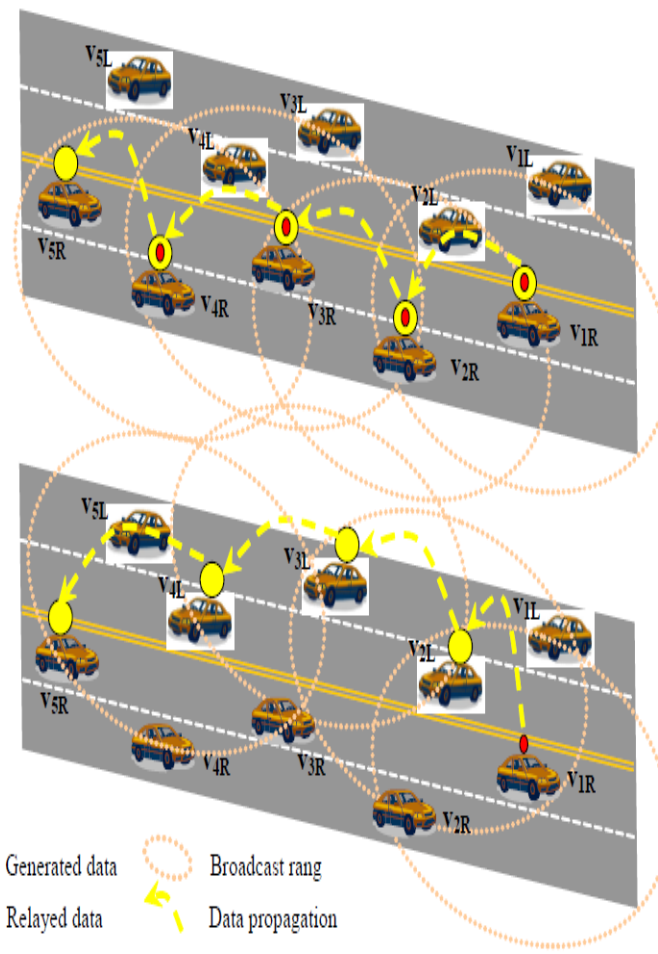

Fig-2

1. If $\mathrm{v} 1$ and $\mathrm{v} 2$ are moving Right, $\mathrm{v} 2$ will accept the packet if $\mathrm{v} 1$ is in front of $\mathrm{v} 2$. This is the case when $\mathrm{v} 1$ broadcasts its generated data.

2. If v1 and v2 aremoving Left, v2 will accept the packet if $\mathrm{v} 2$ is in front of $\mathrm{v} 1$. This is the case when $\mathrm{v} 1$ relays a

packet.

3. If $\mathrm{v} 1$ is moving Right and $\mathrm{v} 2$ is moving Left, $\mathrm{v} 2$ will accept the packet regardless of the relative position of

the vehicles.

Figure 2(b) shows how information is propagated from vehicle v1R to vehicle v5R using opp-dir model. The bidir model combines both same-dir and opp-dir models.

\subsection{Analysis of Dissemination Models}

There was the two main definition in which we can describe the latency time (L) and the broad cats utilization (U). in latency time is defined as the time required to propogated the generated data from the vehicle to vehicle adoc network at $\mathrm{D}$ metre away from it. Broadcast utilization (U) is defined as the percentage of the area covered by the transmission range of broadcast that was not covered by the transmission range of the previous road for the sme packet. We can measue the broadcast utilization by transmission range. To evaluate the performance of our propagation models we consider the following metrics:

- Accuracy: The road in front of each vehicle is divided into regions of 500meters length, and the average error in estimating the position of vehicles in each region is calculated. In the accuracy graphs, the average estimation error for each region is shown, averaged over all the nodes during the simulation.

- Knowledge Percentage: The road in front of each vehicle is divided into regions of 200 meters long. For each region, the percentage of the vehicles in that region about which the current node knows, is defined as the knowledge percentage of that node for that region. The knowledge percentage graph presents the knowledge percentage for each region, averaged over all the nodes during a simulation run.

- Latency Time: This metric measures the elapsed time between the time at which a vehicle's information is generated and time at which it is received by another vehicle. Similar to accuracy metric, the road in front of each vehicle is divided into regions of 500 meters length, and the latency time to receive the information of vehicles in each region is calculated.

- Utilization rate: This metric approximates the broadcast utilization rate described in Section 3. When a vehicle receives a packet, some of the information would not be useful because either they are about cars behind or they are outdated information. Utilization rate of vehicle measures the average percentage of the useful vehicle information contained in received packets by this vehicle. This metric measures the average percentage over all the vehicles in the simulation.

\section{Results}

We experimented with different scenario parameters such as vehicle densities, vehicle speed, and broadcast rate. We also switched between the propagation model where no aggregation mechanism is used and the model in the presence of an aggregation mechanism. However, due to space limitation we limit the results here to the experiments

with different vehicle densities in which the aggregation mechanism is used. For further details about the other experiments, please refer to our technical report [23].

\section{Conclusions and Future Work}

In this paper we presented a formal model of data dissemination in VANETs and how the performance of data dissemination is affected by bi-directional lane mobility. Three models of data dissemination are compared in the context of their performance over the TrafficView system. We show, by means of analysis and simulations, that the data dissemination model that uses 
only vehicles in the opposite direction for propogating data shows best performance. In this paper, we assume that traffic conditions such as density of vehicles in the two opposite directions are similar.

In reality, this would often not be the case. We plan to study the performance of the different disseminationmodels

under such different conditions. In our current system, all cars participate in broadcasting. Our analysis shows that broadcast by a subsection of cars is enough to achieve a good utilization. As future work, we are working on the selection criteria that decides whether a car should participate in broadcasting or not. This criteria will depend on several factors such as traffic density and car speeds. Simulation-based methodologies such as ns-2 use a networking model that is a simplified version of real-life networking. Emulation-based approaches offer interesting tradeoffs between pure simulation and full-scale experiments with acceptable levels of realism and reproducibility[27]. Our future work consists of evaluating the TrafficView system using a wireless grid emulation environment. We are also investigating several other traffic applications that can benefit from the use of the TrafficView dissemination mode.

\section{References}

[1] http://www.quatech.com

[2] http://www.obddiagnostics.com

[3] http://www.its.dot.gov/index.htm

[4]http://www.leearmstrong.com/DSRC/DSRCHomeset.h tm

[5] "Wireless on Wheels," MIT Technology Review, January 2005.

[6] L. Briesemeister, G. Hommel, "Role-based multicast in highly mobile but sparsely connected ad hoc networks," in First Annual Workshop on Mobile Ad Hoc Networking ans Computing, pp. 45-50, Aug. 2000.

[7] Z.D. Chen, H. Kung, and D. Vlah, "Ad Hoc Relay Wireless Networks over Moving Vehicles on Highways," 2nd ACM international symposium on Mobile ad hoc networking and computing (MobiHoc), 2001.

[8] I. Chisalita and N. Shahmehri, "A peer-to-peer approach to vehicular communication for the support of traffic safety applications," 5th IEEE Conference on Intelligent Transportation Systems, 2002.

[9] CORSIM User Manual, Ver. 1.01, The Federal Highway Administration, US Dept. of Transportation, 1996.

[10] S. Dashtinezhad, T. Nadeem, B. Dorohonceanu, C. Borcea, P. Kang, L. Iftode, "TrafficView: A Driver
Assistant Device for Traffic Monitoring based on Car-toCar Communication," Proceedings of the IEEE Semiannual Vehicular Technology Conference, Milan, Italy, May 2004.

[11] General Motors Collaborative Laboratory website available online at http://gm.web.cmu.edu/.

[12] Q. Huang, R. Miller, "The Design of Reliable Protocols for Wireless Traffic Signal Systems”. Technical Report WUCS- 02-45, Washington University, Department of Computer Science and Engineering, St. Louis, MO.

[13] W. Kellerer, "(Auto)Mobile Communication in a Heterogeneous and Converged World," IEEE Personal Communications, Vol. 8(6), pp. 41-47, Dec. 2001.

[14] G. Korkmaz, E. Ekici, F. zgner and U. zgner, "Urban multihop broadcast protocol for inter-vehicle communication systems," 1st ACM international workshop on Vehicular adhoc networks (VANET), 2004.

[15] J. Li, J. Jannotti, D. S. J. De Couto, D. R. Karger, R. Morris, "A Scalable Location Service for Geographic Ad

Hoc Routing”, ACM Mobicom 2000, Boston, MA.

[16] Q. Li, D. Rus, "Sending Messages to Mobile Users in Disconnected Ad-hoc Wireless Networks," ACM Mobicom 2000, Boston, MA.

[17] W. Lou and J. Wu, "On reducing broadcast redundancy in ad hoc wireless networks," IEEE Transactions on Mobile Computing, 2002.

[18] R. Miller and Q. Huang, "An Adaptive Peer-to-Peer Collision Warning System," IEEE Vehicular Technology

Conference (VTC), 2002.

[19] R. Morris, J. Jannotti, F. Kaashoek, J. Li, D. Decouto, "CarNet: A Scalable Ad Hoc Wireless Network System," 9th ACM SIGOPS European Workshop, Kolding, Denmark, Sept. 2000

[20] P. Murphy, E. Welsh, P. Frantz, "Using Bluetooth for Short- Term Ad-Hoc Connections Between Moving Vehicles: A Feasibility Study," IEEE Vehicular Technology Conference (VTC), Birmingham, AL, May 2002.

[21] T. Nadeem, S. Dashtinezhad, C. Liao, L. Iftode, "TrafficView: Traffic Data Dissemination using Car-toCar Communication," ACM Mobile Computing and Communications Review (MC2R), Vol. 8, No. 3, pp. 619, July 2004.

[22] T. Nadeem, S. Dashtinezhad, C. Liao, L. Iftode, "TrafficView: A Scalable Traffic Monitoring System," Proceedings 
of the 2004 IEEE International Conference on Mobile Data Management (MDM), Berkeley, California, USA, January 19-22, 2004. .

[23] T. Nadeem, P. Shankar, L. Iftode, "A Comparative Study of Data Dissemination Models for VANETs," Rutgers University Technical Report, 2006.

[24] Network simulator - ns-2, http://www.isi.edu/nsnam/ns/2.

[25] D. Niculescu, and B. Nath, "Trajectory Based Forwarding and Its Applications," 9th annual international conference on Mobile computing and networking (MobiCom), 2003.

[26] S. Ni, Y. Tseng, Y. Chen, and J. Sheu, "The Broadcast Storm problem in a Mobile Ad Hoc Network," $5^{\text {th }}$ annual international conference on Mobile computing and networking (MobiCom), 1999.

[27] D. Raychaudhuri, I. Seskar, M. Ott, S. Ganu, K. Ramachandran, H. Kremo, R. Siracusa, H. Liu, and M. Singh, "Overview of the ORBIT radio grid testbed for evaluation of next-generation wireless network protocols," IEEE Wireless

Communications and Networking Conference, 2005.

[28] M. Satyanarayanan, "Pervasive Computing: Vision and Challenges," IEEE Personal Communications, Vol. 8(4), pp. 10-17, Aug. 2001.

[29] C. Schwingenschloegl, T. Kosch, "Geocast Enhancements for AODV in Vehicular Networks," ACM Mobile Computing and Communications Review, Vol. 6(3), pp. 96-97, Jul. 2002.

[30] E. Welsh, P. Murphy, P. Frantz, “A Mobile Testbed for GPS-Based ITS/IVC and Ad Hoc Routing Experimentation," International Symposium on Wireless Personal Multimedia Communications (WPMC), Honolulu, HI, Oct. 2002.

[31] H. Wu, R. Fujimoto, R. Guensler, M. Hunter, "MDDV: a mobility-centric data dissemination algorithm for vehicular networks," 1st ACM international workshop on Vehicular ad hoc networks, 2004.

[32] Q. Xu, T. Mak, J. Ko and R. Sengupta, "Vehicletovehicle safety messaging in DSRC," First ACM workshop on Vehicular ad hoc Networks (VANET), 2004 